\title{
Production efficiency of Feshbach molecules in fermion systems
}

\author{
B. E. Dobrescu ${ }^{1}$ and V. L. Pokrovsky ${ }^{1,2}$ \\ ${ }^{1}$ Department of Physics, Texas A\&M University, College Station, Texas 77843-4242 \\ ${ }^{2}$ Landau Institute of Theoretical Physics, Chernogolovka, Moscow region 142432, Russia
}

(Dated: June 6, 2005)

\begin{abstract}
We present a consistent nonequilibrium theory for the production of molecular dimers from a two-component quantum-degenerate atomic Fermi gas, via a linear downward sweep of a magnetic field across a Feshbach resonance. This problem raises interest because it is presently unclear as to why deviations from the universal Landau-Zener formula for the transition probability at twolevel crossing are observed in the experimentally measured production efficiencies. We show that the molecular conversion efficiency is represented by a power series in terms of a dimensionless parameter which, in the zero-temperature limit, depends solely on the initial gas density and the Landau-Zener parameter. Our result reveals a hindrance of the canonical Landau-Zener transition probability due to many-body effects, and presents an explanation for the experimentally observed deviations [K.E. Strecker et al., Phys. Rev. Lett. 91, 080406 (2003)].

PACS numbers: 03.75.Ss, 05.30.Fk
\end{abstract}

The advances of last years in the experimental techniques of atomic and molecular trapping and cooling, combined with the possibility of externally tuning the inter-atomic interactions, have ushered in a series of novel applications: the emergence of a molecular Bose-Einstein condensate (BEC) from a Fermi gas [3, 4], observation of coherent oscillations between an atomic condensate and molecules [5], formation and propagation of matter-wave soliton trains 6], and the examination of Cooper pairing in the BCS-BEC crossover regime [7].

Regardless of their role in further investigations, in all these experiments diatomic molecules are being produced from an atomic BEC or a quantum degenerate two-component Fermi gas either by sweeping a magnetic field across a Feshbach resonance (FR) 8, 9, 10, 11, 13 or by two-photon Raman photo-association 12 . Therefore, the understanding and control of the molecular production mechanism is of special importance.

Notwithstanding the differences in the details of FR experiments, they all show a growth of the molecular conversion efficiency (MCE) with the inverse sweeping rate of the magnetic field, $\dot{B}^{-1}$, that saturates at values less than $100 \%$ in the adiabatic regime.

The attempts aimed at explaining the dependence of MCE on $\dot{B}$, resonance width, initial atomic density and temperature for two-component Fermi systems can be broadly classified into two classes: i)semiphenomenological scenarios [14, 15] that reduce the many-body physics to a two-atom description modeled as a two-state Landau-Zener (LZ) system [1] corresponding, respectively, to the free two-atom scattering state and the bound molecular state, and ii) numerical many-body calculations [16, 19] based on an effective Hamiltonian first proposed by E. Timmermans et al. [17.

Class i) is appealing by its use of simple and intuitive physical pictures, but their predicted (temperature independent) upper MCE limit of $50 \%$ contradicts the experimentally observed far greater values 11,13 . The recent experimental work by E. Hodby et al. 13] also shows a pronounced $T$-dependence of this upper limit. The breakdown of the simple two-level LZ picture can be corrected only by introducing suplimentary ad hoc assumptions in these semi-phenomenological scenarios, whereas it emerges naturally from a bona fide many-body analysis (see below). The work in Class ii) has shown, albeit under some simplifying assumptions, the potential of Hamiltonian [17] in analyzing the temperature dependence of the MCE saturation in the adiabatic regime.

In this Letter we study the atom-molecule conversion in ultra-degenerate two-component Fermi gases subject to a linear downward sweep of a magnetic field across an $s$-wave FR, in the spirit of experiments $9,10,11,13$. We focus on the zero-temperature dynamics, and develop a nonequilibrium theory, pertinent to both weak and strong atom-molecule coupling (measured in Fermi energy units), which allows for a full account of the effects of quantum statistics. The MCE is calculated in terms of real-time Green functions (GF), and represented as a power series in terms of a dimensionless parameter that depends only on the initial gas density and the LZ parameter. An exact evaluation of Feynman-Keldysh diagrams for second and fourth order processes reveals a clear deviation from the LZ transition probability at two-level crossing. This deviation, whose origins reside solely in many-body effects, signals a suppression of the LZ-predicted MCE even for moderately small values of $\dot{B}^{-1}$, as observed experimentally in 10]. Equally important, our MCE result doesn't display an a priori upper limit of $50 \%$ at $T=0$ as suggested in 15 , though further work is necessary to establish the correct limit and its $T$-dependence [13.

The starting point of our analysis is the Hamiltonian [17, 18] describing a system of fermionic atoms FRcoupled to bosonic molecules, $\hat{H}(t)=\hat{H}_{0}(t)+\hat{V}$, with

$$
\hat{H}_{0}(t)=\sum_{\psi=a, b, f} \sum_{\vec{p}} \varepsilon_{\psi}(\vec{p}, t) \hat{\psi}^{\dagger}(\vec{p}) \hat{\psi}(\vec{p}),
$$




$$
\hat{V}=\frac{g}{\sqrt{\mathcal{V}}} \sum_{\vec{p}, \vec{q}}\left[\hat{f}^{\dagger}(\vec{p}+\vec{q}) \hat{b}(\vec{q}) \hat{a}(\vec{p})+\text { h.c. }\right],
$$

where $\hat{a}^{\dagger}(\vec{p}), \hat{a}(\vec{p})$ and $\hat{b}^{\dagger}(\vec{p}), \hat{b}(\vec{p})$ are fermionic creation and annihilation operators describing atoms of momentum $\vec{p}$ and "spins" $\uparrow(a)$ and $\downarrow(b)$, respectively, and $\hat{f}^{\dagger}(\vec{p})$, $\hat{f}(\vec{p})$ play the same role for the bosonic molecules. Other quantities entering $\hat{H}$ are $\varepsilon_{\psi}(\vec{p}, t)=\widetilde{\varepsilon}_{\psi}(\vec{p})-\mu_{\psi} B(t)$, with $\psi=a, b, f$, where $\mu_{\psi}$ is the projection of the magnetic moment along the direction of the magnetic field $B(t)$ with which interacts via Zeeman coupling, and $\widetilde{\varepsilon}_{\psi}(\vec{p})$ is the dispersion relation which accounts for the singleparticle energy renormalization due to nonresonant collisions, and simply reduces to the kinetic energy $p^{2} / 2 m_{\psi}$ in a collisionless regime [20];g is the two-atom-molecule coupling 22] which controls the FR width and $\mathcal{V}$ is the volume of system. The free two-atom scattering state and the molecular state (MS) have different spin configurations and their coupling is mediated via the intra-atomic hyperfine interaction 21] which flips the electronic and nuclear spins of one of the colliding atoms. Depending on the magnetically tuned energy difference between the two states, the MS is quasi-bound (virtual) and belongs to a closed scattering channel if its energy exceeds that of the two-atom channel, becomes resonant with the latter when their energies are equal, and turns truly bound when its energy is the lesser of the two.

In order to probe the MCE dependence on $\dot{B}$ we evaluate real-time GF within the Keldysh formalism (KF) [23]. The method is based on the use of a closed contour for time ordering, which runs from $-\infty$ to $+\infty$ and then back to $-\infty$. Both branches of the contour propagate along the real time axis and any point along them can be characterized by two parameters, written compactly as $\tau^{\gamma}$, with $\tau$ being the time variable and $\gamma$ a bookkeeping index that distinguishes between the forward $(\gamma=+)$ and reverse $(\gamma=-)$ time directions. The basic quantities of $\mathrm{KF}$ are the contour-ordered real-time GF:

$$
i \mathcal{G}^{\alpha \beta}\left(\vec{p}_{1}, \tau_{1} ; \vec{p}_{2}, \tau_{2}\right)=\left\langle\mathbf{T}_{c}\left[\hat{\psi}_{H}\left(\vec{p}_{1}, \tau_{1}^{\alpha}\right) \hat{\psi}_{H}^{\dagger}\left(\vec{p}_{2}, \tau_{2}^{\beta}\right)\right]\right\rangle,
$$

with $\mathcal{G} \equiv \mathcal{A}, \mathcal{B}, \mathcal{F}$ for $\psi=a, b, f$, respectively, $\alpha$, $\beta= \pm$ and $\langle(\cdots)\rangle \equiv \operatorname{Tr}\left[\hat{\rho}\left(t_{0}\right)(\cdots)\right] ; \hat{\rho}\left(t_{0}\right)$ is the initial density operator at $t_{0}=-\infty, \hat{\psi}_{H}$ are the Heisenbergpicture (HP) operators relative to $t_{0}$, and $\mathbf{T}_{c}$ is a contourordering operator. The corresponding free GF read $i \mathcal{G}_{0}^{\alpha \beta}\left(\vec{p}_{1}, \tau_{1} ; \vec{p}_{2}, \tau_{2}\right)=\left\langle\mathbf{T}_{c}\left[\hat{\psi}_{I}\left(\vec{p}_{1}, \tau_{1}^{\alpha}\right) \hat{\psi}_{I}^{\dagger}\left(\vec{p}_{2}, \tau_{2}^{\beta}\right)\right]\right\rangle$, where $\hat{\psi}_{I}$ are the interaction-picture (IP) operators relative to $t_{0}$.

In experiments 9, 10, 11, 13 an ultracold twocomponent Fermi gas is prepared as an incoherent mixture of equal populations in each state, and extreme quantum-degeneracy, at temperatures as low as $T \sim$ $0.05 T_{F}$, has been reached [13], where $T_{F}$ is the Fermi temperature. In this regime the fall-off of the Fermi distribution from 1 to 0 takes place in an extremely narrow energy interval $\sim 0.05 \varepsilon_{F}$, where $\varepsilon_{F}$ is the Fermi energy, and the fuzziness of the Fermi surface becomes virtually unimportant. In this vein [24], we take $\hat{\rho}\left(t_{0}\right)=\left|\Phi_{0}\right\rangle\left\langle\Phi_{0}\right|$, with $\left|\Phi_{0}\right\rangle=\prod \hat{a}^{\dagger}(\vec{p}) \hat{b}^{\dagger}(\vec{p})|\mathrm{VAC}\rangle$, where $|\mathrm{VAC}\rangle$ is the vacuum state [25]. The average number of molecules at time $t$ is given by

$$
\left\langle\hat{N}_{f}\right\rangle(t)=i \sum_{\vec{k}} \mathcal{F}^{+-}(\vec{k}, t ; \vec{k}, t),
$$

and, upon expressing the $\hat{\psi}_{H}$ operators in terms of their IP form $\hat{\psi}_{I}, \hat{\psi}_{H}(t)=\hat{U}_{I}^{\dagger}\left(t, t_{0}\right) \hat{\psi}_{I}(t) \hat{U}_{I}\left(t, t_{0}\right)$, and expanding the IP time-evolution operator $\hat{U}_{I}\left(t, t_{0}\right)$ as a formal series in the coupling constant $g$, the following systematic expansion of $i \mathcal{F}^{+-}(\vec{k}, t ; \vec{k}, t)$ ensues:

$$
\begin{aligned}
& i \mathcal{F}_{0}^{+-}(\vec{k}, t ; \vec{k}, t)+\sum_{n=1}^{\infty}\left(\frac{1}{i \hbar}\right)^{n} \frac{1}{n !} \\
& \times \sum_{\{\gamma\}= \pm}\left(\gamma_{1} \cdots \gamma_{n}\right) \int_{-\infty}^{+\infty} d \tau_{1} \cdots \int_{-\infty}^{+\infty} d \tau_{n} \\
& \times\left\langle\mathbf{T}_{c}\left[\hat{V}_{I}\left(\tau_{1}^{\gamma_{1}}\right) \cdots \hat{V}_{I}\left(\tau_{n}^{\gamma_{n}}\right) \hat{f}_{I}\left(\vec{k}, t^{+}\right) \hat{f}_{I}^{\dagger}\left(\vec{k}, t^{-}\right)\right]\right\rangle,
\end{aligned}
$$

where $\hat{V}_{I}$ is the IP form of $\hat{V}$, and the sum $\sum_{\{\gamma\}= \pm}$ runs over all $n$-tuples $\left(\gamma_{1}, \ldots, \gamma_{n}\right)$ with $\gamma_{j}= \pm$.

Since $\hat{f}_{I}(\vec{p}, t)\left|\Phi_{0}\right\rangle=0$ for any $\vec{p}$ and $t$, and $\hat{V}_{I} \sim \hat{f}_{I}+$ $\hat{f}_{I}^{\dagger}$, it follows that only terms with even $n$ can have a nonvanishing contribution in Eq. (5).

Due to the form of $\left|\Phi_{0}\right\rangle$, the free GF $\mathcal{G}_{0}^{\alpha \beta}\left(\vec{p}_{1}, \tau_{1} ; \vec{p}_{2}, \tau_{2}\right) \quad \propto \quad \delta\left(\vec{p}_{1}, \vec{p}_{2}\right)$, where $\delta\left(\vec{p}_{1}, \vec{p}_{2}\right)$ is the Kronecker delta, and their expressions [26] are

$$
\begin{aligned}
i \mathcal{G}_{0}^{+-}\left(\vec{p} ; \tau_{1}, \tau_{2}\right) & =-\theta\left(\varepsilon_{F}-\widetilde{\varepsilon}_{\psi}(\vec{p})\right) e^{\frac{i}{\hbar} \int_{\tau_{1}}^{\tau_{2}} \varepsilon_{\psi}(\vec{p}, \tau) d \tau}, \\
i \mathcal{G}_{0}^{-+}\left(\vec{p} ; \tau_{1}, \tau_{2}\right) & =\theta\left(\widetilde{\varepsilon}_{\psi}(\vec{p})-\varepsilon_{F}\right) e^{\frac{i}{\hbar} \int_{\tau_{1}}^{\tau_{2}} \varepsilon_{\psi}(\vec{p}, \tau) d \tau},
\end{aligned}
$$

with $\mathcal{G} \equiv \mathcal{A}, \mathcal{B}$ for $\psi=a, b$, respectively, and

$$
\begin{aligned}
& i \mathcal{F}_{0}^{+-}\left(\vec{p} ; \tau_{1}, \tau_{2}\right)=0, \\
& i \mathcal{F}_{0}^{-+}\left(\vec{p} ; \tau_{1}, \tau_{2}\right)=e^{\frac{i}{\hbar} \int_{\tau_{1}}^{\tau_{2}} \varepsilon_{f}(\vec{p}, \tau) d \tau},
\end{aligned}
$$

and finally $\mathcal{G}^{++}\left(\vec{p} ; \tau_{1}, \tau_{2}\right)=\theta(x) \mathcal{G}^{-+}\left(\vec{p} ; \tau_{1}, \tau_{2}\right)+$ $\theta(-x) \mathcal{G}^{+-}\left(\vec{p} ; \tau_{1}, \tau_{2}\right), \quad \mathcal{G}^{--}\left(\vec{p} ; \tau_{1}, \tau_{2}\right)=$ $\theta(x) \mathcal{G}^{+-}\left(\vec{p} ; \tau_{1}, \tau_{2}\right)+\theta(-x) \mathcal{G}^{-+}\left(\vec{p} ; \tau_{1}, \tau_{2}\right)$ for any $\mathcal{G} \equiv \mathcal{A}, \mathcal{B}, \mathcal{F}$, where $x=\tau_{1}-\tau_{2}$ and $\theta(x)$ is the Heaviside function.

Each average corresponding to the terms in Eq. (5) can be performed by means of a generalized version of Wick's theorem in which the contractions are defined with respect to the contour-ordering operator $\mathbf{T}_{c}$, and a diagram is associated with each way of contracting the field operators into pairs 23]. These diagrams have the same topology as those occurring in the ordinary quantum field theory (OQFT) for systems in equilibrium 27], the only difference being an additional label $\gamma= \pm$ that has to 
(D1)

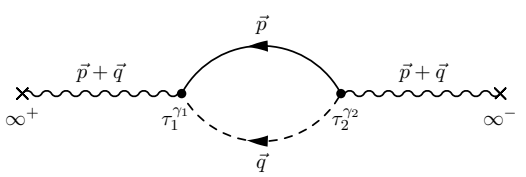

(D2)
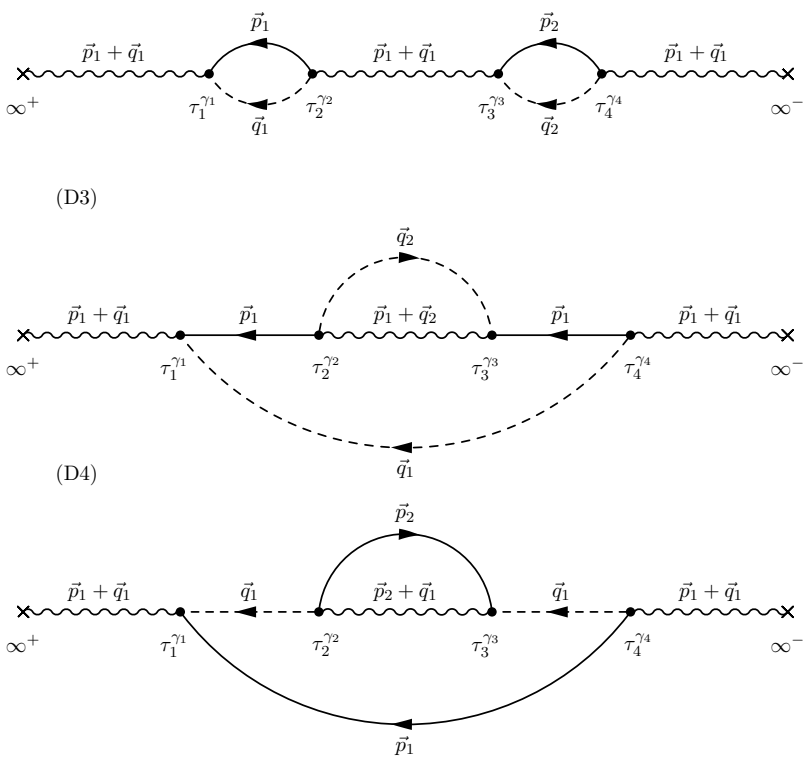

FIG. 1: Feynman-Keldysh diagrams for second (D1) and fourth order (D2 - D4) processes. The free Green functions are represented by continuous lines for $a$-fermions $\left(\mathcal{A}_{0}^{\gamma_{i} \gamma_{j}}\right)$, by dashed lines for $b$-fermions $\left(\mathcal{B}_{0}^{\gamma_{i} \gamma_{j}}\right)$, and by wiggly lines for bosons $\left(\mathcal{F}_{0}^{\gamma_{i} \gamma_{j}}\right)$.

be attached to each interaction vertex. As in OQFT, the disconnected diagrams corresponding to vacuum polarization vanish 23], and only topologically distinct diagrams need to be considered.

Since we are interested in analyzing the dependence of $\mathrm{MCE}$ on $\dot{B}^{-1}$, and not the behavior of the average number of molecules in time, we set the initial time of atomic gas preparation at $t_{0}=-\infty$, and the moleculecounting time at $t_{m}=\infty$. The diagrams representing the contribution from second and fourth order processes to $\left\langle\hat{N}_{f}\right\rangle(\infty)$ are shown in Fig. (11). The (D1) diagrams contribute as

$$
\begin{aligned}
& \left(\frac{g}{\hbar \sqrt{\mathcal{V}}}\right)^{2} \sum_{\vec{p}, \vec{q}} \int_{-\infty}^{+\infty} d \tau_{1} \int_{-\infty}^{+\infty} d \tau_{2} \\
& \times i \mathcal{F}_{0}^{-+}\left(\vec{p}+\vec{q} ; \infty, \tau_{1}\right) i \mathcal{F}_{0}^{-+}\left(\vec{p}+\vec{q} ; \tau_{2}, \infty\right) \\
& \times i \mathcal{A}_{0}^{+-}\left(\vec{p} ; \tau_{1}, \tau_{2}\right) i \mathcal{B}_{0}^{+-}\left(\vec{q} ; \tau_{1}, \tau_{2}\right) \\
& =\frac{N_{0}}{2} \times\left(2 \pi \frac{g^{2}}{\hbar^{2} \dot{\Omega}} \frac{n_{0}}{2}\right)
\end{aligned}
$$

the contribution from the (D2) diagrams is

$$
\begin{aligned}
& -\left(\frac{g}{\hbar \sqrt{\mathcal{V}}}\right)^{4} \sum_{\gamma_{2}, \gamma_{3}= \pm}\left(\gamma_{2} \gamma_{3}\right) \\
& \times \sum_{\vec{p}_{1}, \vec{q}_{1}, \vec{p}_{2}, \vec{q}_{2}} \delta\left(\vec{p}_{1}+\vec{q}_{1}, \vec{p}_{2}+\vec{q}_{2}\right) \\
& \times \int_{-\infty}^{+\infty} d \tau_{1} \cdots \int_{-\infty}^{+\infty} d \tau_{4} i \mathcal{F}_{0}^{++}\left(\vec{p}_{1}+\vec{q}_{1} ; \infty, \tau_{1}\right) \\
& \times i \mathcal{F}_{0}^{\gamma_{2} \gamma_{3}}\left(\vec{p}_{1}+\vec{q}_{1} ; \tau_{2}, \tau_{3}\right) i \mathcal{F}_{0}^{--}\left(\vec{p}_{1}+\vec{q}_{1} ; \tau_{4}, \infty\right) \\
& \times i \mathcal{A}_{0}^{+\gamma_{2}}\left(\vec{p}_{1} ; \tau_{1}, \tau_{2}\right) i \mathcal{B}_{0}^{+\gamma_{2}}\left(\vec{q}_{1} ; \tau_{1}, \tau_{2}\right) \\
& \times i \mathcal{A}_{0}^{\gamma_{3}-}\left(\vec{p}_{2} ; \tau_{3}, \tau_{4}\right) i \mathcal{B}_{0}^{\gamma_{3}-}\left(\vec{q}_{2} ; \tau_{3}, \tau_{4}\right) \\
& =\frac{N_{0}}{2} \times \frac{17}{105}\left(2 \pi \frac{g^{2}}{\hbar^{2} \dot{\Omega}} \frac{n_{0}}{2}\right)^{2},
\end{aligned}
$$

and each of the diagrams (D3) and (D4) contributes equally as

$$
\begin{aligned}
& \left(\frac{g}{\hbar \sqrt{\mathcal{V}}}\right)^{4} \sum_{\gamma_{2}, \gamma_{3}= \pm}\left(\gamma_{2} \gamma_{3}\right) \sum_{\vec{p}_{1}, \vec{q}_{1}} \sum_{\vec{p}_{2}} \\
& \times \int_{-\infty}^{+\infty} d \tau_{1} \cdots \int_{-\infty}^{+\infty} d \tau_{4} i \mathcal{F}_{0}^{++}\left(\vec{p}_{1}+\vec{q}_{1} ; \infty, \tau_{1}\right) \\
& \times i \mathcal{F}_{0}^{\gamma_{2} \gamma_{3}}\left(\vec{p}_{2}+\vec{q}_{1} ; \tau_{2}, \tau_{3}\right) i \mathcal{F}_{0}^{--}\left(\vec{p}_{1}+\vec{q}_{1} ; \tau_{4}, \infty\right) \\
& \times i \mathcal{B}_{0}^{+\gamma_{2}}\left(\vec{q}_{1} ; \tau_{1}, \tau_{2}\right) i \mathcal{B}_{0}^{\gamma_{3}-}\left(\vec{q}_{1} ; \tau_{3}, \tau_{4}\right) \\
& \times i \mathcal{A}_{0}^{+-}\left(\vec{p}_{1} ; \tau_{1}, \tau_{4}\right) i \mathcal{A}_{0}^{\gamma_{3} \gamma_{2}}\left(\vec{p}_{2} ; \tau_{3}, \tau_{2}\right) \\
& =-\frac{N_{0}}{2} \times \frac{1}{2}\left(2 \pi \frac{g^{2}}{\hbar^{2} \dot{\Omega}} \frac{n_{0}}{2}\right)^{2}
\end{aligned}
$$

where $\hbar \dot{\Omega} \equiv\left(\mu_{f}-\mu_{a}-\mu_{b}\right) \dot{B}, N_{0}$ is the total number of atoms present in the system before the magnetic field is applied, and $n_{0}=N_{0} / \mathcal{V}$ is the initial density.

The evaluation of all integrals entering Eqs. (10) - (12) can be carried out exactly, without any supplementary assumptions, and a presentation of the steps involved is beyond the scope of this letter.

Upon collecting results and introducing the notation $\Gamma \equiv 2 \pi \xi_{L Z}\left(\mathcal{V} \frac{n_{0}}{2}\right)$, where $\xi_{L Z}=\frac{g^{2}}{\mathcal{V} \hbar^{2} \dot{\Omega}}$ is the canonical LZ parameter [1], we obtain

$$
\mathrm{MCE}=\frac{2\left\langle\hat{N}_{f}\right\rangle(\infty)}{N_{0}}=\Gamma-\frac{88}{105} \Gamma^{2}+\mathcal{O}\left(\Gamma^{3}\right) .
$$

The $n$-th term of this series is represented by the set of Feynman-Keldysh diagrams containing $2 n$ vertices. A similar approach, i.e. the development of a formal solution in terms of an infinite series in powers of the coupling constant, with further calculation of every term and summation of the resulting algebraic series, has been employed to exactly calculate the probability of nonadiabatic transitions in a multiple-crossing LZ model [2], and it constitutes a powerful alternative to Landau's method [1] of analytic continuation in complex time. 
Eq. (13) reveals deviations from the universal twolevel LZ formula 1], and also from the phenomenological correction proposed in [15] as

$$
\eta\left(1-e^{-\Gamma}\right)=\eta\left(\Gamma-\frac{1}{2} \Gamma^{2}+\mathcal{O}\left(\Gamma^{3}\right)\right),
$$

where $\eta \leq 50 \%$ is a constant that depends on the initial populations of the two-component Fermi gas.

Since $\frac{88}{105}>\frac{1}{2}$, Eq. (13) shows that, as $\dot{B}^{-1}$ increases, the MCE grows slower then predicted by the LZ formula, and this behavior is experimentally supported [10]. In our theory, the approach towards saturation is not due to a mere contraction of the LZ formula by a multiplicative factor determined solely by the initial state preparation, as proposed in the LZ scenarios [14, 15], but has a rather dynamical nature as the atom-molecule conversion takes place in a many-body medium in which the effects of quantum statistics play a crucial role.

Examination of higher order diagrams indicates that MCE is a function depending solely on the parameter $\Gamma$. Therefore, in the extreme adiabatic regime, corresponding to $\Gamma \rightarrow \infty$, MCE must have a universal limit at $T=0$ which, unlike in the phenomenological result (14), is not a priori bounded by $50 \%$. In practice, as the experiments are carried out at finite $T$ and $\Gamma$, the smearing of the
Fermi surface when $T$ approaches $T_{F}$, and the quantum degeneracy reaches its lower limit, must be taken into account 24 for analyzing the $T$-dependence of the MCE saturation [13].

In conclusion, we have considered the MCE for a hyperfine-induced $s$-wave FR and developed a consistent many-body nonequilibrium theory, based on the realtime GF approach, in which all atomic and molecular states are included, and the effects of quantum statistics are fully accounted for.

We demonstrated, by analytically evaluating the MCE up to fourth order in the hyperfine coupling constant, that the canonical LZ formula at two-level crossing is violated in this system due to many-body effects which systematically decrease the LZ transition probability, even for moderately small values of $\dot{B}^{-1}$. This result indicates that in degenerate Fermi gases the effects of quantum statistics near a FR play a crucial role, and the singling out of independent two-atom pairs from an ensemble of delocalized indistinguishable particles, as proposed in the LZ scenarios 14, 15], is untenable.

We acknowledge support by the DOE Grant No. DEFG03-96ER45598, and by NSF under the Grant No. DMR-0321572.
[1] L. Landau, Phys. Z. Sowjetunion 2, 46 (1932); C. Zener, Proc. R. Soc. London, Ser. A 137, 696 (1932).

[2] Y. Kayanuma and S. Fukuchi, J. Phys. B: At. Mol. Phys. 184089 (1985).

[3] M. Greiner et al., Nature (London) 426, 537 (2003).

[4] S. Jochim et al., Science 302, 2101 (2003); M.W. Zwierlein et al., Phys. Rev. Lett. 91, 250401 (2003); T. Bourdel et al., Phys. Rev. Lett. 93, 050401 (2004).

[5] E.A. Donley et al., Nature (London) 417, 529 (2002).

[6] K.E. Strecker et al., Nature (London) 417, 150 (2002).

[7] C.A. Regal et al., Phys. Rev. Lett. 92, 040403 (2004).

[8] J. Herbig et al., Science 301, 1510 (2003); K. Xu et al., Phys. Rev. Lett. 91, 210402 (2003); S. Dürr et al., Phys. Rev. Lett. 92, 020406 (2004).

[9] C.A. Regal et al., Nature (London) 424, 47 (2003).

[10] K.E. Strecker et al., Phys. Rev. Lett. 91, 080406 (2003).

[11] J. Cubizolles et al., Phys. Rev. Lett. 91, 240401 (2003).

[12] R.H. Wynar et al., Science 287, 1016 (2000); C. McKenzie et al., Phys. Rev. Lett. 88, 120403 (2002).

[13] E. Hodby et al., Phys. Rev. Lett. 94, 120402 (2005).

[14] F. H. Mies et al., Phys. Rev. A 61, 022721 (2000).

[15] J. Chwedenczuk et al., Phys. Rev. Lett. 93, 260403 (2004); E. Pazy et al., Phys. Rev. Lett. 93, 120409 (2004).

[16] J. Javanainen et al., Phys. Rev. Lett. 92, 200402 (2004); I. Tikhonenkov et al., cond-mat/0407424 H. Uys et al., cond-mat/0412105

[17] E. Timmermans, K. Furuya, P.W. Milloni, and A.K. Kerman, Phys. Lett. A 285, 228 (2001).

[18] M. Holland, S.J.J.M.F. Kokkelmans, M.L. Chiofalo, and R. Walser, Phys. Rev. Lett. 87, 120406 (2001).
[19] J. E. Williams et al., J. Phys. B 37, L351 (2004).

[20] In our terminology, the term collision refers to scattering processes that cannot alter the number of atoms/molecules.

[21] M. Houbiers et al., Phys. Rev. A 57, R1497 (1998).

[22] $g \sim \epsilon_{h f} a^{3 / 2}$, where $\epsilon_{h f}$ is the strength of the hyperfine interaction responsible for the coupling bewteen the electronic and nuclear spins, and $a$ is the characteristic size of the molecule.

[23] L.V. Keldysh, Zh. Eksp. Teor. Fiz. 47, 1515 (1964); [Sov. Phys. JETP 20, 1018 (1965)]; J. Schwinger, J. Math. Phys. 2, 407 (1961); A.G. Hall, Phys. Lett. B 55, 31 (1975); J. Rammer and H. Smith, Rev. Mod. Phys. 58, 323 (1986); E.M. Lifshitz and L.P. Pitaevskii, Physical Kinetics, Pergamon Press (1981); G.D. Mahan, Manyparticle physics, Plenum Press, NY, 1990.

[24] We leave the extension to $T>0$ for future work.

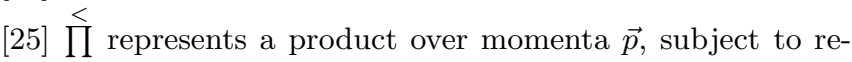
striction $0 \leq \widetilde{\varepsilon}(\vec{p}) \leq \varepsilon_{F}$. Since the two equally populated components of the Fermi gas correspond to two different internal states of the same atom species, we take the same dispersion for them, i.e. $\widetilde{\varepsilon}_{a}(\vec{p})=\widetilde{\varepsilon}_{b}(\vec{p}) \equiv \widetilde{\varepsilon}(\vec{p})$.

[26] Since $\mathcal{G}_{0}^{\alpha \beta}\left(\vec{p}_{1}, \tau_{1} ; \vec{p}_{2}, \tau_{2}\right) \propto \delta\left(\vec{p}_{1}, \vec{p}_{2}\right)$, in order to simplify notation we write $\mathcal{G}_{0}^{\alpha \beta}\left(\vec{p}_{1} ; \tau_{1}, \tau_{2}\right)$ instead.

[27] A.A. Abrikosov, L.P. Gorkov, I.E. Dzyaloshinski, Methods of quantum field theory in statistical physics, Dover, NY, 1963; J.W. Negele and H. Orland, Quantum ManyParticle Systems, Addison-Wesley, 1988. 